\section{Medical Biostatistics, 2nd edition}

Indrayan A. Taylor and Francis Group, Boca Raton, Florida, 2008. Hardcover, 771 pages. ISBN 13-978-1-58488-887-1.

This second edition of Medical Biostatistics provides a comprehensive overview of aspects of research design, evidence-based medicine, and statistical methods for data analysis and presentation.

The book is intended mainly for students, medical and pharmaceutical researchers, health care professionals, and administrators. The material is also intended to be sufficiently comprehensive to be of interest to biostatisticians and their students.

The author's purpose is to present biostatistics (with an emphasis on uncertainty in research and practice) as a medical discipline. To ensure the usefulness of the material for health care professionals, the book emphasizes conceptual understanding, rather than theory or mathematical derivation.

The 21 chapters are organized in a sequence different from that seen in most statistics textbooks. The first 6 chapters focus on uncertainty in medicine, research design, and clinical trial methodology. Chapters 7 to 11 cover various aspects of measurement, variability, and presentation of research data. Chapters 12 to 19 address the core biostatistics topics, such as estimation, testing of statistical hypotheses, survival analysis, and an introduction to multivariable methods. The final 2 chapters deal with a variety of loosely connected topics, including quality of measurement and analysis and fallacies (mainly errors) in research design, data analysis, and interpretation and reporting of results.

The strength of this book is the range of topics, including research design and statistical methods for data analysis, and the fact that it focuses on conceptual understanding. The book contains adequate mathematical explanation, without going beyond the capability or interest of the intended audience. The numerous worked examples are, for the most part, easy to follow, and there is reasonably good use of figures to illustrate concepts. The principal weakness of this book lies in its explanations of fundamental concepts, including probability, confidence intervals, $p$ values, and statistical significance. There are ambiguities and incorrect statements that will confuse or mislead those without an in-depth of knowledge of statistics. For example, the author does not make a clear distinction between frequentist probability (on which this biostatistics text is essentially based) and Bayesian probability (referred to in the text as "personal probability"). This problem pervades explanations for and interpretations of the $p$ value, statistical significance, and confidence intervals. The author draws a distinction between quantitative and qualitative data (by which he means non-interval data) that is not often used elsewhere and which, given the rise in qualitative research methodologies, may also lead to confusion. The book also suffers from insufficient editing, with many grammatical errors that occasionally result in ambiguous or incorrect statements.

Readers with little background in clinical research design might prefer Designing Clinical Research: An Epidemiologic Approach, by Stephen B Hulley and colleagues (Lippincott, Williams \& Wilkins, 2006), or a similarly user-friendly introductory book, Study Design and Statistical Analysis, by Mitchell Katz (Cambridge University Press, 2006).

This book contains much useful information, which could benefit those already familiar with clinical research and the principles of biostatistics. However, during the preparation of an introductory course for PharmD students, I could not use very much of the fundamental material without causing confusion.

Marc Levine, BSc, BScPharm, PhD

Faculty of Pharmaceutical Sciences

University of British Columbia

Vancouver, British Columbia

\section{Managing Pain in the Older Adult}

Jansen M, editor. Springer Publishing Company, New York, New York, 2008. Softcover, 228 pages. ISBN 978-082611567-6.

Managing Pain in the Older Adult, a well-organized review of the issues that arise in treating pain in older patients, highlights a holistic approach to pain management. I was very interested to learn that this text is available, as there are precious few resources available to support clinicians in identifying and appropriately treating pain in older individuals. Although some pharmacists may have trouble agreeing with certain sections, such as the one describing energy therapies, this book does provide a good overview of treatment strategies and could be of benefit to a variety of clinicians.

The strength of this text is its focus on a multimodal approach to developing and implementing an effective pain management plan. The authors start with an effective review of pain theories and the ways in which pain presents in older populations. The effect of persistent pain on the older individual is highlighted with a brief discussion of the types of pain syndromes commonly seen in this population. The authors present an approach that stresses the importance of looking holistically at the patient and taking into account not only the patient's reports of pain but also factors such as function and the psychosocial aspects of the pain. Through the emphasis on a comprehensive approach to pain assessment, we are given insight into a wellorganized, patient-centred strategy for assessment and treatment. 
This approach also allows for mobilization of a full range of treatment strategies, targeting all aspects of pain (physical, psychosocial, functional). To support this approach, the book includes general reviews of pharmacology as well as a review of selected interventional techniques. The latter information is a good general review, but for the pharmacist who is seeking a comprehensive review of the literature regarding medication therapy for persistent pain in older adults, this text falls short. The medication therapies are accurately presented and there is some mention of pharmacokinetic changes that occur in the elderly, but this is done at only a general level. The book does include a general approach to prescribing in older adults, as well as information about the effect of concomitant disease, the World Health Organization pain ladder, and adjuvant treatments.

Where this text distinguishes itself from most other pharmacy texts is in the extensive review of other nonpharmacologic options, including entire chapters on physical therapy and mind-body and energy therapies. The physical therapy section is well written, covering a variety of different approaches and outlining their respective places in therapy. More controversial are the reviews of mind-body and energy therapies. There is an explanation of the theory behind these techniques, and levels of evidence for these strategies are provided. Many patients and some practitioners strongly believe that these strategies are effective, even though it is generally perceived that there is less evidence to support them. The authors suggest that it is important for practitioners to understand these treatments, which their patients may be seeking out. By highlighting the available literature, the authors give readers an opportunity to assess the information and decide the relative merits of the described therapies for themselves.

Managing Pain in the Older Adult is an interesting read that highlights a very good general approach to working with older adults with persistent pain. If read with an analytical eye, it provides information to practitioners looking to enhance their approach to working with older adults suffering from pain.

Allan Mills, PharmD

Department of Pharmacy

Trillium Health Centre

Mississauga, Ontario

\section{Martindale's Drugs Restricted in Sport Pocket Companion, 2008 edition}

Sweetman SC, editor. Pharmaceutical Press, London, UK, 2008. Softcover, 432 pages. ISBN 978085369825 8. Price $£ 19.95$.

According to its editor, Martindale's Drugs Restricted in Sport Pocket Companion, 2008 edition, is "a guide to drugs and medicines that may be restricted in or out of competition in some or all sports, either in their own right, or because they are a derivative of a restricted substance or a member of a prohibited group." The aim of the book is to provide a means for an athlete to determine whether a specific substance is restricted in his or her particular sport.

The book is prefaced with a brief overview of its layout, a disclaimer, a list of abbreviations, and a glossary of terms. The contents are set up alphabetically by drug substance, with the International Nonproprietary Name, where such exists, being used preferentially for each entity. Each monograph is broken down into several sections. Synonyms for each substance are comprehensively listed and extensively indexed in the back of the book for easy reference. There is a separate Cyrillic index. The brief clinical profile that is included with each monograph describes very basic actions and "on-label" therapeutic uses for each substance. The World Anti-Doping Agency (WADA) status and class are listed as well. WADA status refers to whether a drug is prohibited, in or out of competition, and the WADA class refers to the reasoning behind any prohibition.

This reference has been meticulously put together in terms of its comprehensive listing of alternative drug names, and it effectively links that information with the edicts of WADA in terms of prohibited and restricted substances. The multilingual and multinational listings are invaluable, particularly given that athletes' training bases and competition sites span the globe.

The Achilles heel of this book, and any other printed listing of restricted drugs, is that it will be out of date from the moment of publication, since the WADA code is constantly evolving and expanding. Athletes, trainers, coaches, team physicians, and pharmacists cannot assume that consulting a reference such as this one will give them a reliable answer as to whether a substance they may be contemplating taking is restricted for them or not. In particular, one cannot conclude that the absence of a listing for a substance in this publication means that it is not restricted, and even the specific restrictions for substances included in the book may change over time.

For athletes, and for anyone advising athletes, it is reasonable to check Martindale's Drugs Restricted in Sport Pocket Companion as a first step in identifying substances that may result in a positive test. However, without further consultation with national and international sports organizations and their respective doping agencies, such as the Canadian Centre for Ethics in Sport, the Canadian Anti-Doping Program, and WADA, the athlete is at risk of an inadvertent doping violation. For Canadian athletes and their advisors, a visit to the website of the Canadian Centre for Ethics in Sport (http://www.cces.ca) and consultation with their respective sport organizations are both necessary steps when seeking a definitive answer regarding whether a particular substance may result in a doping violation and subsequent sanctioning of the athlete.

Catherine Bond-Mills, BScPhm

London Regional Cancer Program

London Health Sciences Centre

London, Ontario 